\title{
Special Theory of Relativity and the Intrinsicality of Spacetime
}

\section{Shape}

\author{
Erdinç Sayan \\ Middle East Technical University
}

\begin{abstract}
In objection to one of Yuri Balashov's defenses of perdurantism, Matthew Davidson claims that, according to the special theory of relativity, both 3-dimensional and 4-dimensional shapes are nonintrinsic, i.e., they are relative to reference frames. The author argues that 3-dimensional and 4-dimensional spatial shapes are indeed nonintrinsic, but shapes in 3-dimensional and 4-dimensional spacetime are intrinsic according to the special theory of relativity. This follows from the special relativity theory’s claim that spacetime intervals or distances in any n-dimensional spacetime are invariant, unlike spatial distances.
\end{abstract}

Keywords: perdurantism, endurantism, special theory of relativity, spacetime interval, spacetime shape, intrinsic

In his book, Persistence and Spacetime, Yuri Balashov (2010) contends that the endurantist-perdurantist debate in metaphysics must be informed by scientific considerations, in particular, by our knowledge of Einstein's theories of relativity. He calls the endurantist view "3Dism," which is the thesis that objects are wholly present at all times at which they exist, and he calls the perdurantist view "4Dism," which is the thesis that objects are temporally as well as spatially extended. 3Dism claims that objects have three-dimensional spatial parts and spatial shapes at any given instant of their existence, whereas 4Dism claims that objects have four-dimensional spatio-temporal parts and spatio-temporal shapes during their existence. In the language of the special theory of relativity (STR), 4Dism can be described as maintaining that objects are extended in spacetime. In Chapter 8 of his book, Balashov offers a defense of perdurantism that makes use of the notion of shape in Minkowski spacetime.

In a recent note, "Special Relativity and the Intrinsicality of Shape,” Matthew Davidson (2014) argues that, according to STR, three-dimensional shapes of objects aren't intrinsic to them:

... consider two (three or four-dimensional) objects, $\mathrm{O} 1$ and $\mathrm{O} 2$, both moving close to the speed of light at a constant velocity with $\mathrm{O} 1$ moving faster than $\mathrm{O} 2$ relative to a third object, O3. The shape of O3 according to $\mathrm{O} 1$ will differ from the shape of $\mathrm{O} 3$ according to $\mathrm{O} 2$ due to differing amounts of Lorentz contraction. It looks as though we need to relativize three-dimensional shape to reference frames; thus, three-dimensional shape isn’t intrinsic. $(2014,57)$

For this piece of reasoning to be correct, Davidson needs to be talking about the spatial shape of O3, and his conclusion will have to be that three-dimensional spatial shape isn't intrinsic, i.e., such shape is relative to reference frames. But if we are talking about three-dimensional shape in spacetime (with two spatial dimensions plus one time dimension), his conclusion is mistaken according to STR. Three-dimensional

Erdinç Sayan, Prof., Dr., Department of Philosophy, Middle East Technical University, Turkey; main research fields: Analytic Metaphysics, Philosophy of Science, and Philosophy of Physics. Email: esayan@metu.edu.tr. 
spacetime shapes are intrinsic, as we explain in a moment.

Employing a four-dimensional version of his reasoning above, Davidson goes on to argue that four-dimensional shapes are also nonintrinsic. (In the following quotation from him, I add the words in brackets to correct a couple of his important omissions.)

... we can obtain the same result with four-dimensional shape. The situation is as described above, except (i) O1, O2, and $\mathrm{O} 3$ are four-dimensional and (ii) O3 persists for only a minute [in O3’s own reference frame]. O1 and O2 see the entirety of O3's four-dimensional shape by perceiving a series of three-dimensional [spatial] shapes. The four-dimensional shape $\mathrm{O} 1$ "sees" is different than the shape $\mathrm{O} 2$ sees due to differing amounts of Lorentz contraction; the four-dimensional shape will be spatially thinner and will have a longer lifespan for $\mathrm{O} 1$ than for $\mathrm{O} 2$. Thus, it looks as though we also need to relativize four-dimensional shape to reference frames; thus, four-dimensional shape also is not intrinsic. $(2014,58)$

In this argument, when Davidson talks about "four-dimensional shape," he presumably has in mind the shape of an object in the four-dimensional spacetime we live in. But then, once again, his conclusion that four-dimensional spacetime shape is not intrinsic would be false according to STR. Four-dimensional spacetime shapes of objects are intrinsic.

The reason is that, as any textbook treating STR will tell you, spacetime intervals are invariant ("absolute") in STR, i.e., not relative to reference frames-unlike space intervals. In other words, the interval or distance between any two points in spacetime is the same for all observers, ${ }^{1}$ although the distance between two points in space is not. It follows that three-dimensional spacetime shapes of objects are invariant (absolute, intrinsic) too. For it is clear that, if the spacetime distance between any pair of points occupied by an object in spacetime is the same for all observers, then the spacetime shape of the object is the same for all observers. As an analogy, considering the shape of an object, such as a particular chair, in space, if, for every pair of points occupied by the chair in space, the distance between the points is the same for two observers $\mathrm{O} 1$ and O2, then the spatial shape of the chair will be the same for $\mathrm{O} 1$ and O2. (Of course, according to STR, such a situation obtains only when $\mathrm{O} 1$ and $\mathrm{O} 2$ are at rest with respect to each other.)

Davidson's aim is to attack the following view, which he thinks is found in Balashov (1999)²: "[STR] shows that three-dimensional shape isn't intrinsic. But we still can regard four-dimensional shape as intrinsic. Indeed, that we can take four-dimensional shape to be intrinsic is a reason to accept four-dimensional perdurantism” (Davidson 2014, 58). Actually, Balashov has the following statements to make on the issue. According to him, there is

a categorical difference between the nature of 3D shapes of an enduring object... and the nature of the 4D shape of the enduring object's path in spacetime. The 3D shapes (or the having of them) are irreducibly indexed to a frame-relative time, but the $4 \mathrm{D}$ shape is not. ... The $4 \mathrm{D}$ shape is absolute (i.e. had simpliciter) in a way the 3D shapes are not, in the ontology of endurance. (2010, 207; first italics mine)

When Balashov's elliptic term "3D shape” is understood as "3D spatial shape," and his "4D shape” is understood as "4D spacetime shape," it will be clear that the points I made above concord with these remarks of Balashov. $^{3}$

Let us end then with two obvious diagnoses: (i) Davidson seems oblivious to the contrast between the reference-frame relativity of spatial intervals and reference-frame invariance of spacetime intervals in STR. (ii) Davidson does not realize that, when Balashov talks about "3D shape," he means "3D spatial shape;” and when he talks about "4D shape," he means "4D spacetime shape."4 


\section{Notes}

1. The spacetime interval between a pair of events (or points) is given by $(\Delta s)^{2}=(\Delta x)^{2}-(c \Delta t)^{2}$, where $c$ is the speed of light, $\Delta x$ is the difference between space coordinates and $\Delta t$ is the difference between time coordinates of the two events, and "the quantity $\Delta s$, the spacetime interval between two events, is an invariant and has the same value for all inertial observers" (Serway et al. 2005, 33).

2. Davidson mistakenly gives the date of Balashov's 1999 article as 2000.

3. Davidson's above statement of Balashov's position would have been more complete and correct if he had formulated it thus: “[STR] shows that three-dimensional spatial shape isn't intrinsic. But we still can regard four-dimensional spacetime shape as intrinsic. Indeed, that we can take four-dimensional spacetime shape to be intrinsic is a reason to accept four-dimensional perdurantism."

4. I would like to thank Abrek Canbolat and George Darby for their comments and ideas.

\section{Works Cited}

Balashov, Yuri. “Relativistic Objects.” Nous 33 (1999): 644-62.

Balashov, Yuri. Persistence and Spacetime. New York: Oxford University Press, 2010.

Davidson, Matthew. "Special Relativity and the Intrinsicality of Shape.” Analysis 74 (2014): 57-58.

Serway, Raymond A., Clement J. Moses, and Curt A. Moyer. Modern Physics, 3rd ed. Belmont, CA: Thomson Learning, 2005. $<$ http://phy240.ahepl.org/ModPhy-Serway.pdf>. 